\title{
A Prospective Study of Return to Work Across Health Conditions: Perceived Work Attitude, Self-efficacy and Perceived Social Support
}

\author{
Sandra Brouwer • Michiel F. Reneman • \\ Ute Bültmann · Jac J. L. van der Klink • \\ Johan W. Groothoff
}

Published online: 6 November 2009

(c) The Author(s) 2009. This article is published with open access at Springerlink.com

\begin{abstract}
Background The aim of the present study was to conduct subgroup-analyses in a prospective cohort of workers on long-term sickness absence to investigate whether associations between perceived work attitude, selfefficacy and perceived social support and time to RTW differ across different health conditions. Methods The study was based on a sample of 926 workers on sickness absence (6-12 weeks). The participants filled out a baseline questionnaire and were subsequently followed until the tenth month after listing sick. Perceived work attitude was measured with a Dutch language version of the Work Involvement Scale. Perceived social support was measured with a self-constructed standardized scale reflecting a person's perception of social support regarding RTW. Self-efficacy was measured with the standardised Dutch version of the General self-efficacy scale, assessing the subjects' expectations of their general capacities. The sample was divided into three subgroups: musculoskeletal health conditions, other physical health conditions and mental health conditions. Anova analyses and Cox proportional hazards regression analyses were used to identify differences in association between the three factors and the time to RTW
\end{abstract}

S. Brouwer $(\bowtie) \cdot$ U. Bültmann · J. J. L. van der Klink ·

J. W. Groothoff

Department of Health Sciences, Section of Social Medicine,

Work \& Health, University Medical Center Groningen,

University of Groningen, Antonius Deusinglaan 1,

Building 3217, Room 620, 9713 AV Groningen,

The Netherlands

e-mail: sandra.brouwer@med.umcg.nl

M. F. Reneman

Center for Rehabilitation, University Medical Center Groningen,

University of Groningen, PO Box 30002, 9750 RA Haren,

The Netherlands between different subgroups. Results The associations between the perceived work attitude, self-efficacy and perceived social support and the time to RTW vary across different health condition subgroups, not only with regard to the strength of the association but also for the type of factor. In the multivariate model, hazard ratios (HRs) of 1.33 (95\% CI 1.01-1.75) in the musculoskeletal subgroup, and 1.26 (95\% CI 0.89-1.78) in the other physical subgroup were found in perceived work attitude. With regard to perceived social support HRs of 1.39 (95\% CI 1.12-1.99) respectively 1.51 $(1.05-2.17)$ in the same subgroups were found. Only selfefficacy remained in the multivariate model in all subgroups with HRs of 1.49 (95\% CI 1.12-1.99) in the musculoskeletal subgroup, 1.53 (95\% CI 1.07-2.18) in the other physical subgroup and $1.60(1.07-2.40)$ in the mental subgroup. Conclusions The results of this study show that perceived work attitude, self-efficacy and perceived social support are relevant predictors with regard to the time to RTW in all types of health conditions, but that important differences are observed in type of factor and strengths of the relationships between physical and mental health conditions.

Keywords Return-to-work · Long-term sickness . Absence - Work - Perceived work attitude - Self-efficacy · Perceived social support $\cdot$ Subgroup-analyses

\section{Introduction}

Return to work (RTW) can be conceptualized as a complex human behavior change, with the worker taking the final decision to return-to-work [1], influenced by several personal, social and economic factors [1-3]. According to behavioral models, change of behavior is influenced by attitudes (the positive and negative evaluation of the 
expected outcome of a certain behavior), subjective norms (the belief about what others think of the behavior, as derived from the behavior and/or direct feedback of significant others), and self-efficacy, which is generally defined as confidence in being able to carry out a set of specified activities [3, 4]. These behavioral factors have been highlighted in the RTW literature as playing an important role in the RTW process $[1,5]$.

Recently, we have investigated the association between perceived work attitude, self-efficacy and perceived social support and the time to RTW in a prospective one-year follow-up study in workers on long-term sickness absence (6-12 weeks) [6]. The sample consisted of sick listed workers with different types of health conditions. We found that a positive work attitude, high social support, and a high level of self-efficacy were all positively associated with a shorter time to RTW.

It is anticipated that these factors facilitate RTW for almost any health condition [7]. Symptoms and illness may originate from a health condition, but the development of chronicity and disability often depends more on the influence of psychosocial factors [7,8]. The majority of sick-listed workers return to work despite their health condition(s), similarly, symptoms and disability do not necessarily mean incapacity for work [8]. It has also been suggested that especially in non-specific health conditions, like non-specific low back pain, psychosocial factors may play a more important role $[8,9]$ compared to conditions which are 'more severe' in a medical sense. This might entail that perceived work attitude, self-efficacy and perceived social support in a health to health comparison might have different degrees of associations with time to RTW.

Several studies are available that investigate the impact of perceived work attitude, self-efficacy and perceived social support on RTW. However, most of these studies have been performed in a specific symptom group, e.g. musculoskeletal symptoms [7, 10,11], mental disorders [12], cancer [13] or, like in our previous study, in a population of sick-listed workers with different health conditions [1, 6, 14]. Till now, no studies have investigated whether associations between these factors and time to RTW differ across different health conditions. With the present study we want to address a contribution to this gap in disability management research about which factors are relevant for which conditions in terms of impeding and facilitating RTW.

Therefore, the aim of the present study was to conduct subgroup-analyses in a prospective cohort of workers on long-term sickness absence to investigate whether associations between perceived work attitude, self-efficacy and perceived social support and time to RTW differ across different health conditions.

\section{Methods}

Study Sample and Procedure

Data from the prospective 1-year cohort study on 'Return to Work in workers on long-term sickness absence' [15, 16] were used to conduct subgroup-analysis. In 2002, workers on sick leave with different types of symptoms were recruited from Occupational Health Services (OHSs) covering three large regions in the Netherlands [17, 18]. During an inclusion-period of 6 months, 3,918 workers, who were absent for a maximum of 12 weeks and had received a problem analysis (i.e. a Dutch mandatory description of the (dis)abilities of the worker) from their Occupational Physician were sent a letter by the OHS in which they were invited to participate in the study. The letter also explained the purpose and the general outline of the study. The voluntary nature of participation and anonymity of responses was guaranteed. Workers who did not respond within 2 weeks received a written reminder. In total, 1,170 workers (30\%) returned the consent form after which the baseline questionnaire was sent. For all nonrespondents, information on age, gender and region of the OHS was available. A non-response analysis showed that respondents were 2.8 years older than non-respondents (95\% CI 2.16-3.61, $P=0.00$ ), but did not differ according to gender or region of the OHS. The baseline questionnaire was completed by 1,004 (86\%) workers. After completion, 78 workers were excluded from the study for various reasons: 38 workers had not received a problem analysis from their OP or this problem analysis was wrongly administered, 15 workers provided a date of sickness absence that deviated considerably (more than 6 months) from the date provided by the OHSs and 8 workers were on sick leave due to pregnancy-related health symptoms. Because of maternity leave it was not possible to calculate the time to return to work for this group. Five workers had already returned to work before the OHS identified them as possible participants for the study. For nine workers who had returned to work the date of return was not available and three workers were excluded because it was obvious they could not have filled out the questionnaire in a reliable way (e.g. the worker reported he/she did not have the Dutch language skills required). The final sample consisted of 926 workers.

In the present study, these 926 workers were divided into three subgroups, based on the self-reported type of health conditions in the baseline questionnaire as the reason for sickness absence. Categorization was performed based on the International Classification of Functioning, Disability and Health (ICF) Checklist of the WHO part 1a 'Impairments of body functions' and part $1 \mathrm{~b}$ 'Impairments of body structures' [19], with codes added for mental 
conditions such as stress and depression. Workers in the subgroup musculoskeletal conditions suffered from back problems or problems with the upper and lower limbs. The subgroup other physical conditions included workers with diseases of the circulatory, digestive, neurological and respiratory systems. Workers who mentioned conditions such stress, depression or burnout were included in the subgroup with mental health conditions.

\section{Measures}

Socio-demographics (age, gender, educational level, and time to identification by the occupational health service (OHS), type and intensity of health condition and data about the perceived work attitude, self-efficacy and perceived social support were available from the baseline questionnaire which was administered by the workers at baseline, i.e. 6-12 weeks after the onset of sick-leave. Workers were also asked to score the intensity of the conditions on the moment of sick listing on a visual analogue scale ranging from not severe (0) to very severe (100).

Perceived work attitude was measured with a Dutch language version of the Work Involvement Scale (WISDLV) [20], reflecting the degree to which a person wants to be engaged in work. The questionnaire consists of six items; with responses on a 1-4 point scale (strongly disagree, disagree, agree, strongly agree). Higher scores on the WIS-DLV indicate more positive attitude towards work. The internal consistency (Cronbach alpha) of the WIS-DLV in this cohort study was 0.67 .

Perceived social support was measured with a selfconstructed standardized scale reflecting a person's perception of social support from family, friends, supervisor and co-workers, care-givers and community regarding RTW. The scale includes 12 items; each item is preceded by the question "How much support did you receive during your period of sickness from..." with responses on a 1-4 point scale (no support, little support, much support or not applicable). Higher scores indicate more perceived social support. The internal consistency (Cronbach alpha) of perceived social support was 0.75 .

Self-efficacy was measured with the standardised Dutch version of the General self-efficacy scale [21], assessing the subjects' expectations of their general capacities [22]. This 16-item questionnaire incorporated three subscales: willingness to expend effort in completing the behavior, persistence in the face of adversity, and willingness to initiate behavior. It consists of five response items (ranging form disagree to agree); higher scores indicate higher selfefficacy. The internal consistency (Cronbach alpha) was 0.80 for willingness to expend effort in completing the behavior, 0.70 for the persistence in the face of adversity, and 0.73 for the willingness to initiate behavior scale.

Follow-up questionnaires were filled out 10 months after listing sick. RTW was measured by two questions. Firstly, workers had to indicate their current work status: full RTW, partial RTW or being on full sick leave. Full RTW was defined as working the same number of hours as in the initial work contract. Secondly, workers who indicated to have returned to work had to write down the exact RTW date. If the respondent had not written down the RTW date or the respondent was lost to follow up, the RTW date of the OHSs was used as a proxy for calculating the time to RTW.

\section{Data Analysis}

Kruskal-Wallis one-way ANOVA analyses were performed to detect significant differences between the three health condition subgroups. Kaplan-Meier survival analyses and Cox proportional hazards regression analyses were used to model the effect of the independent variables on time to RTW, which was defined as the time between sickness absence identification by the OHS and first full RTW. All analyses were conducted for each subgroup separately. The Kaplan-Meier survival analysis was performed to calculate the median time to RTW for each subgroup. Prognostic variables were dichotomized into "low" and "high" based on the median split [7]. For all variables the low group was used as reference group. A HR higher than 1 reflects a shorter duration of sickness absence when compared to the reference group.

With respect to the univariate and multivariate analyses, the Cox's proportional hazards model was used to study the association between the perceived work attitude, self-efficacy and perceived social support and the time to RTW. Age, gender, level of education, time to identification by the OHS, and intensity of conditions were included as control variables. First, the relationship between all prognostic factors and the outcome was assessed. For the multivariate regression analysis those factors which were statistical significant at the $P \leq 0.15$ level in the univariate analyses were included in the model and adjusted for the five control variables. Next, variables were omitted by backward selection, depending on their level of statistical significance $(P \leq 0.10)$. Subsequently, we separately added the potential variables to the multivariate model which were not statistically significant in the univariate analysis to determine their association with the outcome measure in the presence of other prognostic factors. The proportional hazards assumption was graphically checked by plotting the "log minus log" survivor function. All analyses were performed with SPSS for Windows 14.0 [17]. 


\section{Results}

Baseline Characteristics

In total, 862 workers were included in the subgroup-analysis: 352 workers were categorized in the musculoskeletal condition subgroup, 265 workers in the other physical conditions subgroup, and 245 workers in the mental health condition' subgroup. Workers $(n=35)$ who mentioned multiple conditions which could fall into more than one health condition subgroup were excluded from subgroupanalyses. Other workers $(n=20)$ mentioned fatigue as the only symptom for which they had reported absent. Because fatigue can (might) be related to all three health conditions, these workers were also excluded. Nine workers did not mention the type of health condition and were therefore excluded.

The socio-demographic characteristics of the study subsamples are presented in Table 1. Significant differences between the three subgroups were found for age, gender, and educational level. The mental health condition subgroup was the youngest subgroup, with the lowest percentage of males and the highest percentage of high educational level. No significant differences were found in time to identification by the OHS and intensity of conditions.
After 10 month follow-up, the percentage of workers who fully returned to work was not significantly different $(P=0.18)$ across the different subgroups. However, with regard to the time to return to work workers with musculoskeletal conditions showed the shortest median time to RTW, while workers with other physical conditions had the longest median time to RTW $(P=0.01$, Table 1 ; Fig. 1).

Perceived Work Attitude, Self-efficacy and Perceived Social Support

Workers with musculoskeletal conditions reported significantly higher levels of perceived work attitude, self-efficacy and perceived social support compared to the other physical condition subgroup and the mental health condition subgroup (see Table 1). The results of the uni- and multivariate analyses for the three subgroups are presented in Table 2. In the univariate analysis of the musculoskeletal condition subgroup, perceived work attitude, perceived social support and two subscales of self-efficacy-willingness to expend effort in completing a behavior and willingness to initiate a behavior-were significantly associated $(P \leq 0.15)$ with time to RTW. After applying the backward selection procedure, three factors remained in the multivariate model: perceived work attitude (HR 1.33,

Table 1 Characteristics of the subgroup samples

\begin{tabular}{|c|c|c|c|c|}
\hline Variables $^{\mathrm{a}}$ & $\begin{array}{l}\text { Musculoskeletal } \\
\text { conditions } \\
(n=342-352)\end{array}$ & $\begin{array}{l}\text { Other physical } \\
\text { health conditions } \\
(n=251-265)\end{array}$ & $\begin{array}{l}\text { Mental health } \\
\text { conditions } \\
(n=238-245)\end{array}$ & $P$-value \\
\hline Age [mean (SD)] & $45.4(9.4)$ & $47.7(9.5)$ & $44.2(9.4)$ & $<0.01$ \\
\hline $\operatorname{Sex}(\%$ men $)$ & 57 & 56 & 38 & $<0.01$ \\
\hline Educational level (\%) & & & & $<0.01$ \\
\hline Very low & 8 & 10 & 6 & \\
\hline Low & 47 & 27 & 20 & \\
\hline Medium & 28 & 27 & 34 & \\
\hline High & 17 & 35 & 40 & \\
\hline Time to identification by OHS (in days, SD) & $56.4(20.5)$ & $58.6(20.8)$ & $55.8(19.1)$ & 0.29 \\
\hline Intensity of conditions $(0-100)$ & $75.5(18.2)$ & $72.5(22.1)$ & $76.8(15.0)$ & 0.45 \\
\hline Full return to work at 10 month follow-up (\%) & 68 & 60 & 65 & 0.18 \\
\hline Time to return to work in days (median, IQR) & $111.00(48.00-215.00)$ & $165.00(64.50-226.00)$ & $160.00(72.50-223.00)$ & 0.01 \\
\hline Time to return to work in days [mean (SD)] & $126.85(85.70)$ & $148.31(85.00)$ & $148.78(82.34)$ & 0.01 \\
\hline Work attitude [mean (SD)] & $19.39(3.02)$ & $18.74(3.59)$ & $18.46(3.35)$ & $<0.01$ \\
\hline Social support [mean $(\mathrm{SD})]$ & $20.98(5.01)$ & $19.34(4.56)$ & $19.71(4.33)$ & $<0.01$ \\
\hline \multicolumn{5}{|l|}{ Self-efficacy [mean (SD)] } \\
\hline Willingness to expend effort in completing a behavior & $24.86(4.53)$ & $23.40(5.07)$ & $19.97(5.60)$ & $<0.01$ \\
\hline Willingness to initiate behavior & $15.40(3.71)$ & $14.97(3.94)$ & $13.43(4.23)$ & $<0.01$ \\
\hline Persistence in the face of adversity & $25.52(3.73)$ & $24.93(3.86)$ & $23.23(4.52)$ & $<0.01$ \\
\hline
\end{tabular}

a Time to return to work in days: log rank test; return to work, gender, and educational level: chi-square; all other variables: ANOVA 




Fig. 1 Kaplan-Meier curves: cumulative percentage of RTW in musculoskeletal-, other physical- and mental health condition subgroups

95\% CI 1.01-1.75, $P=0.04)$, perceived social support (HR 1.39, 95\% CI 1.12-1.99, $P=0.02$ ) and willingness to expend effort in completing the behavior (HR $1.49,95 \%$ CI 1.12-1.99, $P<0.01)$.

In the univariate analysis of the other physical condition subgroup perceived work attitude, perceived social support and one subscale of self-efficacy-willingness to expend effort in completing the behavior-were significantly associated $(P \leq 0.15)$ with the time to RTW. After applying the backward selection procedure, three factors remained in the multivariate model, two of the factors were significantly associated with the time to RTW $(P<0.05)$ : perceived social support (HR 1.51, 95\% CI 1.05-2.17, $P=0.03$ ) and willingness to expend effort in completing the behavior (HR 1.53, 95\% CI 1.07-2.18, $P=0.02$ ).

In the univariate analysis of the mental health condition subgroup, one subscale of self-efficacy-willingness to expend effort in completing the behavior-was significantly associated $(P \leq 0.15)$ with the time to RTW (HR $1.49,95 \%$ CI $1.01-2.18, P=0.04)$. After applying the backward selection procedure, this factor remained in the multivariate model (HR 1.60, 95\% CI 1.07-2.40, $P=0.02$ ). Separately adding those variables to the multivariate model which were not significant in the univariate analysis did not result in the inclusion of other factors in all of the three subgroups.

\section{Discussion and Conclusion}

This study shows that the association between perceived work attitude, self-efficacy and perceived social support and time to RTW differ across different health conditions.
Firstly, significant differences were found on the mean scores of perceived work attitude, self-efficacy and perceived social support between the three subgroups (i.e. musculoskeletal, other physical and mental), with the highest mean scores in the musculoskeletal subgroup. Secondly, differences were observed with respect to the strength of the associations (HR) in both the uni- and multivariate model between perceived work attitude and perceived social support and time to RTW, especially when comparing the HRs of musculoskeletal condition and other physical condition with the mental health condition subgroup. Previous analyses of the total sample [6] showed that a better perceived work attitude and more perceived social support are statistically significant factors for time to RTW and that the estimated hazard (HR) of a shorter time to RTW is 1.19 times higher in individuals who reported a higher work attitude and 1.12 times higher in individuals who reported more social support compared to the reference group. In the subgroup analyses these effects of perceived work attitude and perceived social support can only be seen and are even slightly more beneficial in the subgroup of workers with musculoskeletal diseases (adjusted $\mathrm{HR}=1.33$ respectively $\mathrm{HR}=1.39$ ) or other physical health problems (adjusted HR $=1.26$ respectively $\mathrm{HR}=1.51$ ). However, in the subgroup with mental health problems better perceived work attitude and more perceived social support are actually a barrier to RTW $(\mathrm{HR}=0.94$ respectively HR $=0.80)$. For work attitude this might be conceivable because many mental health conditions may originate in a too high workload and people that are dedicated to their work might be at risk to overload and exhaust themselves. Social support knows many forms and the perception of the support is even more important than the actual support [18]. The effective form of instrumental social support might be more easily administered in physical health conditions in the form of work adjustments than in mental health conditions. These findings show that it may be beneficial to intervene on perceived work attitudes and perceived social support among workers with physical health problems, but it may be counterproductive to do so among workers with mental health problems.

The subscale of self-efficacy "Willingness to expend effort in performing a specific behaviour" was the only factor, which was significantly associated with the time to RTW in all three subgroups in both the uni- and multivariate models. This result emphasizes the role of self-efficacy in the RTW process as described by other authors $[1,3,5]$. Up to now, however, a comprehensive picture of the role of selfefficacy in the development and duration of work disability and insight in the effectiveness of interventions addressing self-efficacy to facilitate RTW are still lacking.

In the present study suggestive evidence has been pointed to the existence of an important difference between 





physical and mental health condition in accordance to the relevance of perceived work attitude, self-efficacy and perceived social support as predictors for time to RTW. A possible explanation for the differences between the physical and mental subgroups might be that the RTW process needs to be conceptualized as a complex, developmental phenomenon that is influenced by a multitude of factors acting at different points in time after the onset of symptoms [2, 23-25]. According to our results, these factors might be strongly associated with the time to RTW in the physical condition groups 10 months after the onset of sick-leave, whereas in the mental subgroups stronger associations exist at a different point in time after the onset of sickness absence. The cyclic patterns of symptoms might be different for musculoskeletal conditions (which might remit within weeks), whereas mental health symptoms might require a much longer period of time to remit. Since these conditions have elements of chronicity, the client's prior experience with his or her symptoms will likely play a role in how he or she foresees return to work.

Therefore, the natural history of the different health conditions may contribute to the understanding of its impact on how perceived work attitude, self-efficacy and perceived social support will predict outcomes. Krause et al. [2] confirmed in their study that the strength of the association of psychosocial factors with the time to RTW varies with progressing phases of disability. These time-dependent influences may have been responsible for the present findings. Due to our research design, we could not monitor these stages or phases in the RTW process, because data were only available at baseline and 10 months follow-up. This may have lead to the masking of the effects of specific factors [7, 26]. Future research should be designed with more repeated measurements to get better insight into the RTW process. Along with these repeated measurements, more indepth qualitative studies might be needed, including all relevant stakeholders, to learn more about the RTW process.

An alternative explanation for the differences between the three subgroups might be the strength of correlation between the type of health condition and the prognostic factor. Low self-efficacy has previously been shown to be highly correlated with high levels of avoidance behavior, depression and emotional distress [27]. While we did not measure these psychological factors, it cannot simply be assumed that the impact of these psychological factors will differ between subgroups. However, it might be presumed that these psychological factors will be more present in the mental health subgroup that indeed had the lowest rates of self-efficacy. Further research is needed to explore whether there are differences in relationships between perceived work attitude, self-efficacy and perceived social support and psychological factors between different health condition groups.
The different subgroup profiles that were found may have clinical relevance in the development of diseasegroup-specific disability management guidelines or may be beneficial to develop disease-specific interventions. In accordance to the differences in natural history of the different health conditions further phase-specific research is needed to explore the variation in strength of the association of perceived work attitude, self-efficacy and perceived social support with the time to RTW between different health condition groups. Such knowledge might be helpful to improve the effectiveness of treatment, because suboptimal timing of interventions may result in disappointing results $[28,29]$. These issues should be considered before recommendations for intervention are made.

Although we expected that severity of symptoms may differ across the subgroups as well, no significant differences were found. These findings might be in line with a study of Dasinger et al. [30], who found that the severity of injury had a significantly stronger effect on RTW during the first 30 days post injury, but not after 30 days post injury. Our study population of absent workers due to different health conditions was included 6-12 weeks after the onset of sick-leave.

It should be noted that this is a first explorative study focusing on the prospective associations between perceived work attitude, self-efficacy and perceived social support and the time to RTW in subgroups across different health conditions. Other studies have conducted subgroup-analyses within one health condition group, especially in low back pain patients [31-33]. The subgroups used in the present study were comparable to subgroups used in other studies $[34,35]$. In the present study, the categorization into subgroups was based on the self-reported type of health condition. Unfortunately, no access to the computerized files of the OHS was given; therefore we could not use the diagnosis (disease-code) of each worker as filled out by a physician based on ICD codes. Furthermore, there might be some misclassification of subjects with respect to health condition subgroups. The health conditions as reported by the workers were coded with the help of the ICF-checklist. However, the classification of symptoms is always subject to interpretation and to keep misclassification to a minimum, the material was thoroughly checked and subjects which could not be classified clearly were excluded from the analyses.

The results of this study show that perceived work attitude, self-efficacy and perceived social support and the time to RTW are relevant in all types of health conditions, but that important differences are observed in type of factor and strengths of the relationships between physical and mental health conditions. This has not yet been shown in any prospective study before. Future research should focus on subgroup analyses to get more insight into the influence 
of the type of health-condition as a moderator on the association between these investigated factors and the time to RTW. Moreover, a further exploration of the time-specific variation in (strength of the) associations between perceived work attitude, self-efficacy and perceived social support and the time to RTW in the different health condition subgroups during several stages in the RTW process is warranted.

Open Access This article is distributed under the terms of the Creative Commons Attribution Noncommercial License which permits any noncommercial use, distribution, and reproduction in any medium, provided the original author(s) and source are credited.

\section{References}

1. Labriola M, Lund T, Christensen KB, Albertsen K, Bültmann U, Jensen JN, et al. Does self-efficacy predict return-to-work after sickness absence? A prospective study among 930 workers with sickness absence for three weeks or more. Work. 2007;29:233-8.

2. Krause N, Frank JW, Dasinger LK, Sullivan MJ, Sinclair SJ. Factors of duration of disability and return-to-work after workrelated injury and illness: challenges for future research. Am J Ind Med. 2001;40:464-84.

3. Franche R-L, Krause N. Readiness for return to work following injury or illness: conceptualizing the interpersonal impact of health care, workplace, and insurance factors. J Occup Rehabil. 2002;12:233-56.

4. Ajzen I. The theory of planned behavior. Organ Behav Hum Decis Process. 1991;50:179-211.

5. Shaw WS, Huang YH. Concerns and expectations about returning to work with low back pain: identifying themes from focus groups and semi-structured interviews. Disabil Rehabil. 2005;27(21): 1269-81.

6. Brouwer S, Krol B, Reneman MF, Bültmann U, Franche RL, van der Klink JJL, et al. Behavioral factors as predictors of return to work after long-term sickness absence: An application of the Theory of Planned Behavior. J Occup Rehab. 2009;19(2):166-74.

7. Krause N, Dasinger LK, Deegan LJ, Rudolph L, Brand RJ. Psychosocial job factors and return-to-work after compensated low back injury: a disability phase-specific analysis. Am J Ind Med. 2001;40:374-92.

8. Waddell G. Preventing incapacity in people with musculoskeletal disorders. Br Med Bull. 2006;1-15.

9. Barsky AJ, Borus JF. Functional somatic syndromes. Ann Intern Med. 1999;130:910-21.

10. Heymans MW, de Vet HCW, Knol DL, Bongers PM, Koes BW, van Mechelen W. Workers' beliefs and expectations affect return to work over 12 months. J Occup Rehabil. 2006;16:685-95.

11. Lötters F, Franche RL, Hogg-Johnson S, Burdorf A, Pole JD. The prognostic value of depressive symptoms, fear-avoidance, and self-efficacy for duration of lost-time benefit in workers with musculoskeletal disorders. J Occup Environ Med. 2006; 63(12):794-801.

12. van Oostrum SH, Anema JR, Terluin B, Venema A, de Vet HCW, van Mechelen W. Development of a workplace intervention for sick-listed workers with stress-related mental disorders: intervention mapping as a useful tool. BMC Health Ser Res. 2007;7:127.

13. Hirai K, Suzuki Y, Tsuneto S, Ikenaga M, Hosaka T, Kashiwagi T. A structural model of the relationships among self-efficacy, psychological adjustment, and physical condition in Japanese advanced cancer patients. Psycho Oncol. 2002;11:221-9.

14. Heibel B, Josephson M, Jensen I, Stark S, Vingard E. Return to work expectation predicts work in chronic musculoskeletal and behavioral health disorders: prospective study with clinical implications. J Occup Rehabil. 2006;16:173-84.

15. Post M, Krol B, Groothoff JW. Work-related factors of return to work of workers on long-term sickness absence. Disabil Rehabil. 2005;27(9):481-8.

16. Post M, Krol B, Groothoff JW. Health-related factors of return to work of workers on long-term sickness absence. Disabil Rehabil. 2006;28(5):289-97.

17. SPSS advanced models 14.0. Chicago, IL: SPSS; 2005.

18. Gallo JJ. The effect of social support on depression in caregivers of the elderly. J Fam Pract. 1990;30(4):430-40.

19. International classification of functioning, disability and health. Geneva: World Health Organisation; 2001.

20. Warr P, Cook J, Wall T. Scales for the measurement of some work attitude and aspects of psychological well-being. J Occup Psychol. 1979;52:129-48.

21. Bosscher RJ, Smit JH, Kempen GIJM. Algemene competentieverwachtingen bij ouderen; een onderzoek naar de psychometrische kenmerken van de Algemene Competentieschaal (ALCOS). Ned Tijdschr Psychol. 1997;52:239-48. [In Dutch; general competency expectations in elderly; a study into the psychometric properties of the general competency scale].

22. Scherer M, Maddux JE, Mercandante B, Prentice-Dunn S, Jacobs B, Rogers RW. The self-efficacy scale: construction and validation. Psychol Rep. 1982;51:663-71.

23. Krause N, Ragland DR. Occupational disability due to low back pain: a new interdisciplinary classification based on a phase model of disability. Spine. 1994;19(9):1011-20.

24. Frank JW, Brooker AS, DeMaio SE, Kerr MS, Maetzel A, Shannon HS, et al. Disability resulting from occupational low back pain. Part II: what do we know about secondary prevention? A review of the scientific evidence on prevention after disability begins. Spine. 1996;21(24):2918-29.

25. Frank JW, Kerr MS, Brooker AS, DeMaio SE, Maetzel A, Shannon HS, et al. Disability resulting from occupational low back pain. Part I: what do we know about primary prevention? A review of the scientific evidence on prevention before disability begins. Spine. 1996;21(24):2908-17.

26. Oleinick A, Gluck JV, Guire K. Factors affecting first return to work following a compensable occupational back injury. Am J Ind Med. 1996;30(5):540-55.

27. Arnstein P, Caudill M, Mandle C, Norris A, Beasley R. Selfefficacy as a mediator of the relationship between pain intensity, disability and depression in chronic pain patients. Pain. 1999;80: 483-91.

28. Sinclair SJ, Hogg-Johnson SH, Mondloch MV, Shields SA. The effectiveness of an early active intervention program for workers with soft-tissue injuries. The early claimant cohort study. Spine. 1997;22(24):2919-31.

29. Frank J, Sinclair S, Hogg-Johnson S. et al. Preventing disability from work-related low-back pain. New evidence gives new hope-if we can just get all the players onside. Can Med Assoc J. 1998;158:1625-31.

30. Dasinger LK, Krause N, Deegan LJ, Brand JB, Rudolph L. Physical workplace factors and return to work after compensated low back injury: a disability phase-specific analysis. J Occup Environ Med. 2000;42(3):323-33.

31. Denison E, Åsenlöf P, Sandborgh M, Lindberg P. Musculoskeletal pain in primary health care: subgroups based on pain intensity, disability, self-efficacy, and fear-avoidance variables. J Pain. 2007;8(1):67-74. 
32. Steenstra IA, Knol DL, Bongers PM, Anema JR, van Mechelen $\mathrm{W}$, de Vet HC. What works best for whom? An exploratory, subgroup analysis in a randomized, controlled trial on the effectiveness of a workplace intervention in low back pain patients on return to work. Spine. 2009;20(12):1243-9.

33. Turk D, Okifuji A, Sinclair J, Starz T. Differential responses by psychosocial subgroups of fibromyalgia syndrome patients to an interdisciplinary treatment. Arthritis Care Res. 1998;11:397-404.
34. Reiso H, Nygard JF, Brage S, Gulbrandsen P, Tellnes G. Work ability and duration of certified sickness absence. Scand J Public Health. 2001;29:218-25.

35. Janssen N, Schröer CAP, Nijhuis FJN. The natural course of fatigue in a working population. Maastricht: Datawyse; 2004. 